\title{
Corrigendum
}

\section{Inverse mean value property of harmonic functions}

\author{
W. Hansen', I. Netuka ${ }^{2}$ \\ ${ }^{1}$ Fakultät für Mathematik, Universität Bielefeld, D-33501 Bielefeld, Germany \\ ${ }^{2}$ Mathematical Institute, Charles University, Sokolovská 83, CZ-18600 Praha, Czech Repub- \\ lic
}

Math. Ann. 297, 147-156 (1993)

Received: 26 January 1995

Mathematics Subject Classification (1991): 31A05, 31B05, 31D05

Let $\lambda$ denote Lebesgue measure on $\mathbb{R}^{d}, d \geqq 2$, and for every Lebesgue measurable subset $A$ of $\mathbb{R}^{d}$ such that $0<\lambda(A)<\infty$ let $\lambda_{A}$ denote the normalized Lebesgue measure on $A$, i.e., $\lambda_{A}=(\lambda(A))^{-1} 1_{A} \lambda$. Moreover define

$$
G(z)= \begin{cases}-\log |z| & \text { when } d=2 \\ |z|^{2-d} & \text { when } d>2\end{cases}
$$

Our main result in [1] is the following:

Theorem. Let $A$ be a Lebesgue measurable subset of $\mathbb{R}^{d}, d \geqq 2$, such that $0<\lambda(A)<\infty$ and let $B$ denote the open ball of centre 0 such that $\lambda(B)=$ $\lambda(A)$. Then $\lambda_{A}(h)=h(0)$ holds for every bounded $h=G^{1} C^{\lambda}-G^{1} \lambda, C$ and $D$ compact sets contained in $C A$, if and only if $\lambda(B \backslash A)=0$.

The proof is based on Lemma 2.1 where we claimed the existence of a constant $c>0$ such that the following holds: For every unit vector $\theta \in \mathbb{R}^{d}$, all $a, b \in \mathbb{R}$ satisfying $0<a<b<\sqrt{2} a$ and every compact subset $K$ of $C B_{b}$, there exist neighborhoods $V$ and $W$ of $x=a \theta$ and $y=b \theta$, respectively, such that for all probability measures $\mu$ on $V$ and $v$ on $W$ the function

$$
h= \begin{cases}\frac{1}{b / a-1}\left(G^{\nu}-G^{\nu}(0)-\left(G^{\mu}-G^{\mu}(0)\right)\right) & \text { when } d=2 \\ \frac{1}{b / a-1}\left(G^{\nu} / G^{\nu}(0)-\left(G^{\mu} / G^{\mu}(0)\right)\right) & \text { when } d>2\end{cases}
$$

satisfies $h<c$ on $\overline{B_{a}}, h(z)>(1 / c)(a /\|z\|)^{d-2}$ for every $z \in K$ and

$$
\int_{\{h<0\} \backslash B_{a}}|h| d \lambda<c a^{d} \sqrt{b / a-1} .
$$


We are indebted to $D$. Singman for having pointed out that, contrary to our statement, the functions $h$ have no upper bound on $\bar{B}_{a}$ if $d>2$. Using continuity properties it was sufficient to consider the special case $\mu=\varepsilon_{x}$ and $v=\varepsilon_{y}$. Unfortunately, the inequality $g(z) \leqq g(-x)$ at the beginning of the proof of the lemma is erroneous.

If $d=2$ we know, however, that

$$
h:=\frac{1}{b / a-1}\left(\left(G^{\varepsilon_{y}}-G^{\varepsilon_{y}}(0)-\left(G^{\varepsilon_{x}}-G^{\varepsilon_{x}}(0)\right)\right)<1\right.
$$

on $\bar{B}_{a}$ since obviously $G^{\varepsilon_{y}}-G^{\varepsilon_{x}}<0$ on $\bar{B}_{a}$ and

$$
\frac{G^{\varepsilon_{x}}(0)-G^{\varepsilon_{y}}(0)}{b / a-1}=\frac{\ln (b / a)}{b / a-1}<1 .
$$

So for $d=2$ the proof of the theorem itself needs no modification.

If $d>2$ we have

$$
\begin{aligned}
h & :=\frac{1}{b / a-1}\left(G^{\varepsilon_{y}} / G^{\varepsilon_{y}}(0)-G^{\varepsilon_{x}} / G^{\varepsilon_{x}}(0)\right) \\
& =\frac{1}{b / a-1}\left[\frac{G^{\varepsilon_{y}}-G^{\varepsilon_{x}}}{G^{\varepsilon_{x}}(0)}+\left(\frac{1}{G^{\varepsilon_{y}}(0)}-\frac{1}{G^{\varepsilon_{x}}(0)}\right) G^{\varepsilon_{y}}\right]
\end{aligned}
$$

where again $G^{a_{y}} \leqq G^{\varepsilon_{x}}$ on $\bar{B}_{a}$. Moreover,

$$
\begin{aligned}
\frac{1}{b / a-1}\left(\frac{1}{G^{\varepsilon_{y}}(0)}-\frac{1}{G^{\varepsilon_{x}}(0)}\right) & =\frac{b^{d-2}-a^{d-2}}{b / a-1} \\
& =a^{d-2} \frac{(b / a)^{d-2}-1}{b / a-1} \leqq(d-2)(\sqrt{2})^{d-3} a^{d-2} .
\end{aligned}
$$

So in Lemma 2.1 we should have written that $h<c a^{d-2} G^{\varepsilon_{x}}$ on $\bar{B}_{a}$.

It is easy to get the necessary modification in the proof of our theorem for $d>2$ : Assume that $\lambda(B \backslash A)>0$, but $\lambda_{A}(h)=h(0)$ for every $h=G^{\lambda} C$ with $C$ being a compact subset of $[A$. As in [1] define

$$
r:=\sup \left\{\rho \geqq 0: \lambda\left(B_{\rho} \backslash \dot{A}\right)=0\right\} .
$$

Let us note first that $r>0$. Indeed, suppose the contrary and fix a point $z$ in the boundary of $B$. Obviously,

$$
\int_{A \backslash B} G^{\varepsilon_{0}} d \lambda<\lambda(A \backslash B) G^{\varepsilon_{0}}(z)=\lambda(B \backslash A) G^{\varepsilon_{0}}(z)<\int_{B \backslash A} G^{\varepsilon_{0}} d \lambda .
$$

Adding $\int_{A \cap B} G^{\varepsilon_{0}} d \lambda$ we obtain that $\int_{A} G^{\varepsilon_{0}} d \lambda<\int_{B} G^{\varepsilon_{0}} d \lambda$, hence $G^{\lambda_{A}}(0)<$ $G^{\lambda_{B}}(0)$ (recall that $\lambda(A)=\lambda(B)$ ). By continuity there exists $\delta>0$ such that $G^{\lambda_{A}}<G^{\lambda_{B}}$ on $B_{\delta}$. Having assumed that $r=0$ there exists a compact subset $C$ of $B_{\delta} \backslash A$ such that $\lambda(C)>0$. Taking $f=G^{\lambda_{C}}$ we then know that $\lambda_{A}(f)=$ $\lambda_{C}\left(G^{\lambda_{A}}\right)<\lambda_{C}\left(G^{\lambda_{B}}\right)=\lambda_{B}(f)$. On the other hand $\lambda_{A}(f)=f(0) \geqq \lambda_{B}(f)$ since $f$ is superharmonic. This contradiction shows that $r>0$. 
We now choose $K, \eta, \varepsilon, a, b, \mu, v$ as in [1]. Considering $h:=(b / a-1)\left(G^{v} /\right.$ $\left.G^{v}(0)-G^{\mu} / G^{\mu}(0)\right)$ we obtain that

$$
0=\int_{A} h d \lambda-\int_{B_{a}} h d \lambda=\int_{A \backslash B_{a}} h d \lambda-\int_{B_{a} \backslash A} h d \lambda .
$$

Since $h<c a^{d-2} G^{\varepsilon_{x}}$ on $\bar{B}_{a}$ we have

$$
\begin{aligned}
\int_{B_{a} \backslash A} h d \lambda & \leqq \int_{B_{a} \backslash B_{r}} h^{+} d \lambda \\
& \leqq c a^{d-2} \int_{B_{a} \backslash B_{r}} G^{\varepsilon_{x}} d \lambda \leqq c(r+\varepsilon)^{d-2} \int_{B_{r+\varepsilon} \backslash B_{r}} G^{\varepsilon_{x}} d \lambda .
\end{aligned}
$$

Note that $\int_{B_{r+\varepsilon} \backslash B_{r}} G^{\varepsilon_{x}} d \lambda$ is as small as we want since the functions $1_{B_{r+1}} G^{\varepsilon y}$, $y \in \mathbb{R}^{d}$, are uniformly integrable. Furthermore

$$
\int_{A \backslash B_{a}} h d \lambda \geqq \int_{K} h d \lambda-\int_{\{h<0\} \backslash B_{a}}|h| d \lambda \geqq \eta r^{d-2}-c(r+\varepsilon)^{d} \sqrt{\varepsilon} .
$$

So we conclude that $\int_{B_{a} \backslash A} h d \lambda<\int_{A \backslash B_{a}} h d \lambda$ if $\varepsilon$ is sufficiently small. This contradiction shows that $\lambda(B \backslash A)=0$ finishing the proof.

\section{Reference}

1. Hansen, W., Netuka, I.: Inverse mean value property of harmonic functions, Math. Ann. 297 (1993), 147-156 